\title{
The influence of the catalyst on the kinetics of ethyl metacrylate synthesis
}

\author{
Mirosław Grzesik ${ }^{1}$, Teresa Witczak ${ }^{2}$ \\ 1 Institute of Chemical Engineering, Polish Academy of Sciences, ul. Battycka 5, 44-100 Gliwice, Poland, \\ rrgrzesi@cyf-kr.edu.pl, fax (+48 12) 6624761 \\ 2 Faculty of Food Technology, University of Agriculture, ul. Balicka 122, 30-149 Kraków, Poland
}

\begin{abstract}
The synthesis of ethyl metacrylate in the liquid phase was studied. Tungstophosphoric and molybdophosphoric acids, which belong to heteropolyacids group, were used as a catalyst. The chemical compounds from this group are often utilized in the catalysis with regard to their activity and selectivity. The rate equations, reaction rate constants and equilibrium constants have been determined. The reaction order and the kinetic parameters of the kinetic relations were determined by the integral method. All rate equations are formulated with activities taking the non ideal effects of the compounds into consideration. It was found that the kinetics of the esterification of the presented reactions was non-elementary.
\end{abstract}

Keywords: esterification, heteropoliacids, kinetics.

This work has been dedicated to Professor Fryderyk Stręk on the occasion of the 80th anniversary of his birthday.

\section{INTRODUCTION}

The ethyl methacrylate is an ester of methacrylic acid and ethyl alcohol. It is used in numerous organic syntheses to obtain various polymers and copolymers. With regard to its properties it has found a wide application in the production of resins, synthetic rubber and paints ${ }^{1}$.

The direct esterification of acid with an alcohol can be described by the following stoichiometric equation:

$\mathrm{CH}_{2}=\mathrm{C}\left(\mathrm{CH}_{3}\right)-\mathrm{COOH}+\mathrm{C}_{2} \mathrm{H}_{5} \mathrm{OH} \Leftrightarrow \mathrm{CH}_{2}=\mathrm{C}\left(\mathrm{CH}_{3}\right)$ - $\mathrm{COOC}_{2} \mathrm{H}_{5}+\mathrm{H}_{2} \mathrm{O}$

The important problem in the esterification of methacrylic acid are the side reactions of polymerization (polymerization of methacrylic acid, polymerization of ester). In order to avoid them, the polymerization inhibitors are added to the reaction mixture.

The most common catalysts, used in industrial synthesis of ethyl methacrylate, are strong mineral acids (hydrochloric, sulphuric). Despite the fact that they act very well as catalysts, their toxicity, corrosivity, and problems connected with the creation of side-products dangerous for natural environment forced scientists to search for other compounds that would be free of the mentioned above drawbacks.

Since the eighties of the last century, in industrial processes based on acid catalysis (e.g. hydration of alkenes), new compounds - that are part of the heteropolyacids group - have found an application. The highest activity and selectivity in acid catalysis and in catalytic oxidation was demonstrated by heteropolyacids with a Keggin structure, and mainly they were subjected to an in-depth research ${ }^{2}$. The research has shown that they could be used in both: the homogenic and in the heterogenic systems ${ }^{3-7}$. In the works of Izumi ${ }^{3}$ and Kozhevnikov ${ }^{7}$ the authors made an attempt to describe the mechanism of a homogenic reaction catalyzed with a heteropolyacid. They found that in addition to a participating proton, a heteropoly anion also participates in the process, which, in turn, additively increases the reaction rate in comparison to the reactions conducted in the presence of traditionally-used acidic catalysts. Because of that, heteropolyacids are interesting compounds that can find application in novel technologies.
This work examines the kinetics of ethyl methacrylate synthesis in the presence of tungstophosphoric acid and molybdophosphoric acid. The influence of temperature, catalyst concentration and the molar ratio of reagents on the reaction rate, were evaluated.

\section{EXPERIMENTAL}

The experiments were carried out in an isothermal batch reactor $(100 \mathrm{ml})$ equipped with a thermometer $( \pm 0.1 \mathrm{~K})$, a sampling cock and an azeotropic head with a reflux condenser. A magnetic stirrer (1000 rpm) was used to ensure the homogeneity of the reaction mixture. The reactor was placed in a heating jacket fed in a closed cycle with a liquid from an ultrathermostat.

The experiment began with placing the weighted amounts of alcohol, hydroquinone and catalyst in the reactor. Then the mixture was heated to the temperature at which the reaction was to be conducted and methacrylic acid was added. Immediately after the temperature restabilised, the first sample for the analysis was collected. The time intervals at which the concentration of the reaction mixture was measured were appropriately extended with the decreasing reaction rate. The part of studies intended to obtain the equilibrium data was carried out until the minimum changes in the reagent concentrations were observed. Moreover, basing on the chromatographic analyses of the randomly selected samples, only minimal amounts of reaction by-products were discovered. Such way of treatment unequivocally allowed to conclude, that only the esterification reaction took place in the system, and the determined change of acid concentration (by $\mathrm{KOH}$ titration and calculating acid number) depicted the real reaction progress.

In the experiments the following parameters were examined: reaction temperature, the molar ratios of the reagents and catalyst concentration in order to determine their influence on the degree of the conversion of the methacrylic acid. The temperature was changed in a range from 338 to $358 \mathrm{~K}$ for the tungstophosphoric acid and from 343 to $353 \mathrm{~K}$ for molybdophosphoric acid, the molar ratio of acid to ethyl alcohol from 1:3 to 1:10; the catalyst concentration in the range from 4.92 to $14.76 \mathrm{wt} \%$ for the 
tungstophosphoric acid, and from 3.12 to 12.48 wt $\%$ for molybdophosphoric acid.

\section{RESULTS AND DISCUSSION}

Using the values of acid numbers, the degree of conversion for methacrylic acid was calculated from the formula:

$\alpha_{K}(t)=1-\frac{L K(t) \cdot m_{0}}{n_{0 K} \cdot 56000}, t \in\left[t_{0}, t_{f}\right]$

where $n_{0 K}$ is the initial mole number of methacrylic acid, $m_{0}$ is the total mass of reagents in the reactor, $L K(t)$ is the acid number after the time $t$ and 56000 is an analytical factor.

The gained set of results $\alpha_{\mathrm{K}}=\mathrm{f}(\mathrm{t})$, was employed to establish kinetic equations by the integral method.

A preliminary analysis of the experimental results indicated that the reaction rate did not depend on the concentration of the reagents in a simple manner. As the chemical process under study took place in the liquid phase and the mixtures were multicomponent and contained polar substances, it was supposed that the reaction rate would be proportional not to the concentration, but to the activity of the reagents. Therefore, activity coefficients were determined by using the UNIFAC (Universal Functional Group Activity Coefficients) method. From the results it was found that the activity of the reagents did not correspond with their concentration. Accordingly, the kinetic models developed were based on concentration activities.

For both the tungstophosphoric and molybdophosphoric acid, the same, non-elementary, form of the kinetic equation was obtained:

$r_{K}=k \cdot c_{c a t}\left(\frac{a_{K} \cdot a_{A}}{a_{E}^{0.3}}-\frac{a_{E}^{0.7} \cdot a_{W}}{K_{a}}\right)$

where $K_{a}$ is thermodynamic equilibrium constant, $k$ is reaction rate constant, $a_{K}, a_{A}, a_{E}, a_{W}$ are activities of acid, alcohol, ester and water, respectively.

Thermodynamic equilibrium constant was also reagents activity dependent. Its change as a function of temperature is described by the relationship below:

$K_{a}=2.114 \cdot 10^{3} \exp \left(\frac{-16400}{R T}\right)$

Using reaction rate constants at different temperatures the frequency coefficient $\left(k_{0}\right)$ and activation energy $(E)$ of the investigated reaction were calculated from the Arrhenius equation by means of linear regression. Their values are shown in Table 1.

Based on the derived model equations, the degree of conversion of methacrylic acid, was established and compared with the values obtained experimentally. The results of fitting the calculated results to the experimental data are shown in Figs 1, and 2. Only selected results are given.

Table 1. Kinetic parameters

\begin{tabular}{|l|c|c|}
\hline CATALYST & $\begin{array}{c}E \\
{\left[\mathrm{~J} \mathrm{~mol}^{-1}\right]}\end{array}$ & $\begin{array}{c}k_{0} \\
{\left[\mathrm{~m}^{5.1} \mathrm{~mol}^{-1.7} \mathrm{~s}^{-1}\right]}\end{array}$ \\
\hline $\mathrm{H}_{3} \mathrm{PW}_{12} \mathrm{O}_{40}$ & $72000 \pm 500$ & 84.33 \\
\hline $\mathrm{H}_{3} \mathrm{PMo}_{12} \mathrm{O}_{40}$ & $72200 \pm 600$ & 99.67 \\
\hline
\end{tabular}

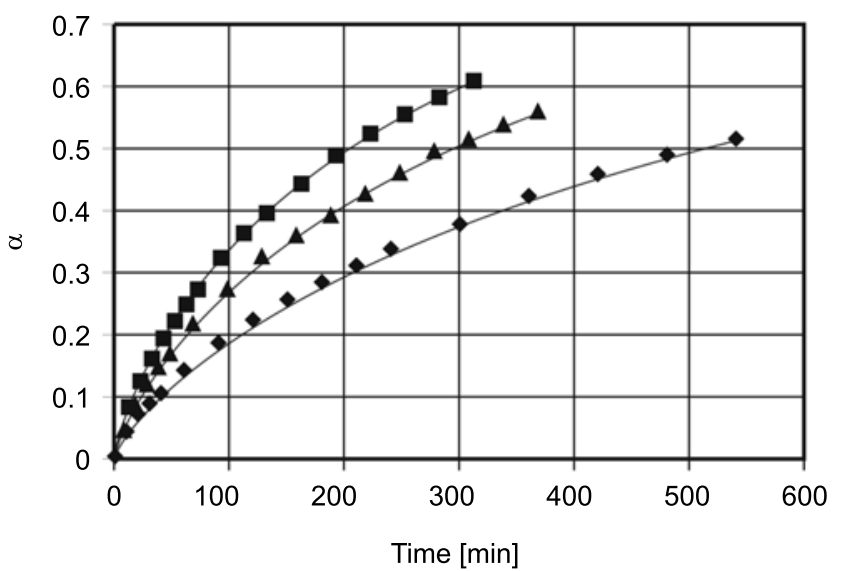

Figure 1. Comparison of computer results (solid lines) with the experimental data (symbols) for the esterification reaction of methacrylic acid with ethanol in the presence of $\mathrm{H}_{3} \mathrm{PW}_{12} \mathrm{O}_{40}(\bullet \mathrm{T}=348 \mathrm{~K} \quad 1: 54.92$ $\mathrm{wt} \%, \Delta \mathrm{T}=348 \mathrm{~K} 1: 58.94 \mathrm{wt} \%, \boldsymbol{\mathrm { T }}=348 \mathrm{~K} 1: 514.76$ $\mathrm{wt} \%)$

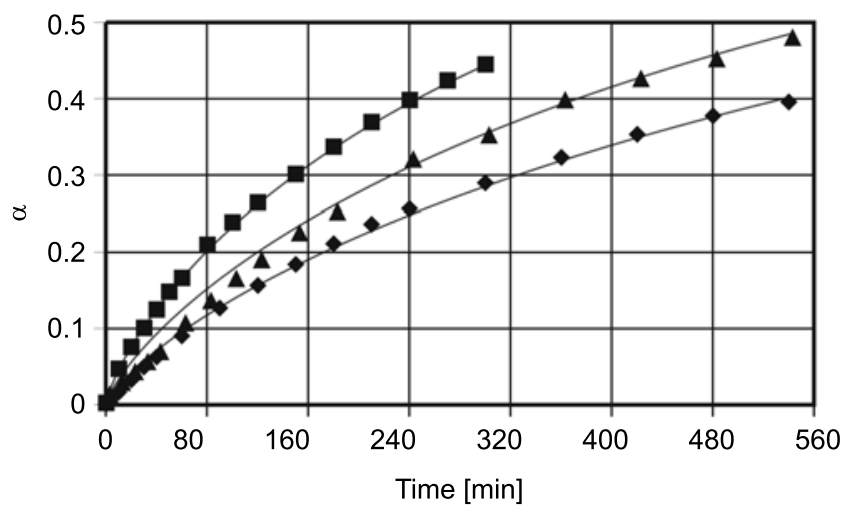

Figure 2. Comparison of computer results (solid lines) with the experimental data (symbols) for the esterification reaction of methacrylic acid with ethanol in the presence of $\mathrm{H}_{3} \mathrm{PMo}_{12} \mathrm{O}_{40}(\bullet \mathrm{T}=348 \mathrm{~K} 1: 33.12$ $\mathrm{wt} \%, \Delta \mathrm{T}=348 \mathrm{~K} 1: 36.24 \mathrm{wt} \%,-\mathrm{T}=348 \mathrm{~K} 1: 312.48$ $\mathrm{wt} \%)$

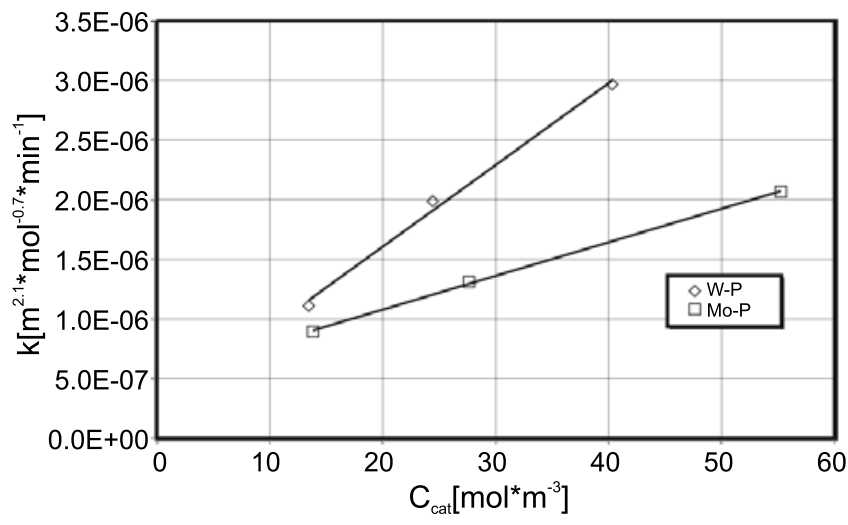

Figure 3. The effect of catalyst concentration on the reaction rate constant

\section{CONCLUSIONS}

The tungstophosphoric and molybdophosphoric acids turned out to be active catalysts of the reaction of esterification of methacrylic acid using ethanol. Under the conditions of the process, it was possible to obtain a relatively high reaction rate. The relationship between the reaction rate constant and catalyst concentration is shown in Fig. 3. 
The higher values of the reaction rate constant for the reaction catalyzed by tungstophosphoric acid were revealed in a higher activity of this catalyst. It was also confirmed by the time needed to convert half of the methacrylic acid, which was shorter for the reaction carried out in the presence of tungstophosphoric acid.

Based on the results shown in the work of Witczak et al. ${ }^{8}$ one can compare heteropolyacids with a typical catalyst of esterification conducted in a homogenous system - the sulphuric acid. In both cases it was not successful to obtain elementary kinetic. Additionally, these rate equations differed in a reaction order. In the case of sulphuric acid it was the fourth order, but for heteropolyacids there was a fractional value established (1.5). In all the cases the activation energies were comparable to one another (for

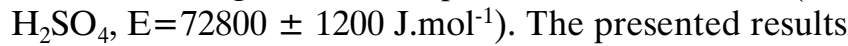
of kinetic experiments have shown that heteropolyacids can be used in industrial processes of direct synthesis of ethyl methacrylate and become competitive for traditional esterification catalysts.

\section{LITERATURE CITED}

(1) Lipińska-Łuczyn E., Perkowski J.: Analiza rynku monomerów metakrylowych, Przemysł Chemiczny, 1998, 77(10), 363.

(2) Izumi Y., Urabe K., Onaka M.: Zeolite, Clay and Heteropoly Acid in Organic Reactions Kodansha/VCH, Tokyo, 1992.

(3) Izumi Y.: Hydration/Hydrolysis by Solid Acids, Catalysis Today, 1997, 33, 371.

(4) Kozhevnikov I. V.: Heteropoly Acids and Related Compounds as Catalysts for Fine Chemical Synthesis, Catalysis Reviews - Science Engineering, 1995, 37(2), 311.

(5) Chen X., Xu Z., Okuhara T.: Liquid Phase Esterification of Acrylic Acid with 1-Butanol Catalyzed by Solid Acid Catalysts, Applied Catalysis, 1999, 180, 261

(6) Izumi Y., Hisano K., Hida T.: Acid Catalysis of Silicaincluded Heteropolyacid in Polar Reaction Media, Applied Catalysis, 1999, 181, 277.

(7) Hu Ch., Hashimoto M., Okuhara T., Misono M.: Catalysis by Heteropoly Compounds .XXII. Reactions of Esters and Esterification Catalyzed by Heteropolyacids in a Homogeneous Liquid-Phase Effects of the Central Atom of Heteropolyanions Having Tungsten as the Addenda Atom, Journal of Catalysis, 1993, 143, 437.

(8) Witczak M., Grzesik M., Skrzypek J.: Kinetyka estryfikacji kwasu metakrylowego niższymi alkoholami alifatycznymi, Inżynieria Chemiczna i Procesowa, 2004, 25 (2), 321. 